\title{
The inner disks of EXor-type eruptive stars
}

\author{
Nikoletta Sipos ${ }^{1}$ and Ágnes Kóspál ${ }^{2}$ \\ ${ }^{1}$ Institute for Astronomy, ETH Zürich, Wolfgang-Pauli-Strasse 27, 8093 Zurich, Switzerland \\ email: niki@phys.ethz.ch \\ ${ }^{2}$ Research and Scientific Support Department, European Space Agency (ESA, ESTEC, \\ SRE-SA), PO Box 299, 2200 AG, Noordwijk, The Netherlands, email: akospal@rssd.esa.int
}

\begin{abstract}
EX Lupi-type young stars (EXors) show sporadic brightenings of several magnitudes, caused by the episodic increase in the accretion rate of the circumstellar matter onto the young star. As the inner disk plays a crucial role during the onset of the outburst, we examined the quiescent properties of the circumstellar environment of EXors, focusing on the inner regions. We found that in case of three EXors (VY Tau, V1143 Ori and EX Lup) the spectral energy distributions show no or weak excess above the stellar photosphere at NIR-MIR wavelengths, indicative of inner disk clearing. A detailed radiative transfer modeling of the sources revealed that the inner regions of these disks had to go through significant evolution, either the inner radius of the dusty disk is beyond the sublimation radius and/or the inner disks are flattened.
\end{abstract}

Keywords. stars: formation - stars: protoplanetary disks - stars: individual: VY Tau, V1143 Ori, EX Lup

\section{Introduction}

EXors are young low-mass stars, showing sudden flare-ups separated by intervals of quiescent periods (Herbig 1989). Outbursts typically last for a couple of months or a year, and correspond to an optical brightening of 1-5 magnitudes, while between two eruptions they spend several years in a low-state. This type of eruptive phenomena is associated with a significant enhancement in mass accretion rates (up to $10^{-5} \mathrm{M}_{\odot} / \mathrm{yr}$ ). Eruptions are important periods for building up stellar mass, and they influence the structure and the composition of the disk (e.g. Mosoni et al. 2013, Ábrahám et al. 2009). The circumstellar environment and especially the regions closest to the star must play an essential role in driving the eruptions. We examine the dusty circumstellar environment of three EXors (VY Tau, V1143 Ori and EX Lup) in quiescence, looking for features that might explain their eruptive nature and set them apart from normal T Tauri stars.

\section{EXors with weak NIR-MIR excess}

We compared the spectral energy distributions (SEDs) of the EXors to those of normal T Tauri stars in the NIR-MIR regime. For the comparison we used the T Tauri median SED (Fig. 1). We show here three members of the group, that all have a weak or missing excess over the stellar photosphere compared to typical T Tauri stars at the NIR domain. They run below the lower quartile of the Taurus median at short wavelengths. At longer wavelengths they gradually reach it and EX Lup also exceeds the upper quartile. This characteristic in general might be indicative of an inner disk clearing behind the dust sublimation radius or the settling of the inner disk (Furlan et al. 2006, Luhman et al. 2010). We also examined five other EXors (XZ Tau, DR Tau, NY Ori, UZ Tau and V1118 Ori), but they have a significant excess above the photosphere at all wavelengths, implying the presence of an optically thick disk already at small radii. 

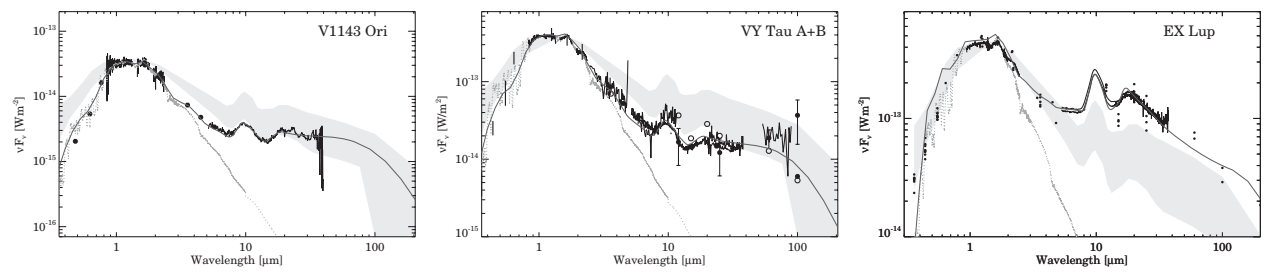

Figure 1. SEDs of V1143 Ori, VY Tau and EX Lup. The light grey band marks the upper and lower quartile of the median SED of Taurus young stars (D'Alessio et al. 1999, Furlan et al. 2006). The dotted line shows the stellar photosphere of each source. The solid line shows our best fit models (Sipos et al. (2009), Sipos \& Kóspál (in prep.)).

Table 1. Overview of stellar properties and distances of the sources.

\begin{tabular}{lcccccc}
\hline Object & Spectral type & $T_{\text {star }}(\mathrm{K})$ & $M_{\text {star }}\left(\mathrm{M}_{\odot}\right)$ & $L_{\text {star }}\left(\mathrm{L}_{\odot}\right)$ & $A_{V}(\mathrm{mag})$ & Distance $(\mathrm{pc})$ \\
\hline VY Tau A/B & M0/M2 & $3800 / 3370$ & $0.85 / 0.35$ & $0.26 / 0.07$ & 0.48 & 145 \\
V1143 Ori & M2 & 3500 & 0.5 & 0.2 & 0 & 400 \\
EX Lup & M0 & 3800 & 0.6 & 0.48 & 0 & 155 \\
\hline
\end{tabular}

We tested what kind of geometry may cause the reduced flux at short wavelengths for EX Lup, V1143 Ori and VY Tau by modeling their SEDs with the help of the radiative transfer code RADMC (Dullemond \& Dominik 2004). The best fit models are shown in Fig. 1, the basic properties of the sources are summarized in Table 1. For the details of the modeling, the applied geometry and the resulting parameters we refer to Sipos et al. (2009) and Sipos \& Kóspál (in prep.), here we point out our findings in connection with the innermost part of the dusty disks. In the case of EX Lup the dusty disk starts at radii $\sim 3.5$ times the sublimation radius, beyond that the disk can be characterized with parameters typical of normal T Tauri stars. V1143 Ori also has a dust free inner hole that stretches $\sim 3$ times behind the sublimation radius, and the scale height of the inner disk is reduced by $\sim 80 \%$ compared to a typical value. In order to fit the SED of VY Tau we either had to use an enlarged inner hole or a reduction of the scale height by $60 \%$.

Three out of eight EXors are surrounded by dust free inner holes that stretch further from the central star than the sublimation radius, and in two cases the disks have a reduced scale height, probably due to grain growth or settling. The inner clearing is a surprising result given the high accretion rates during the repetitive eruptions. According to certain outburst scenarios one would expect a massive reservoir in the inner disk that feeds the accretion during the outburst. The enlarged hole can be the result of the eruptions emptying the inner disk, photoevaporation or an unknown small companion.

\section{References}

Ábrahám, P., Juhász, A., Dullemond, C. P., et al. 2009, Nature, 459, 224

D'Alessio, P., Calvet, N., Hartmann, L., Lizano, S., \& Cantó, J. 1999, ApJ, 527, 893

Dullemond, C. P. \& Dominik, C. 2004, A\&A, 417, 159

Furlan, E., Hartmann, L., Calvet, N., et al. 2006, ApJS, 165, 568

Herbig, G. H. 1989, ESO Conference and Workshop Proceedings, 33, 233

Luhman, K. L., Allen, P. R., Espaillat, C., Hartmann, L., \& Calvet, N. 2010, ApJS, 186, 111

Mosoni, L., Sipos, N., Ábrahám, P., et al. 2013, A\&3A, 552, A62

Sipos, N., Ábrahám, P., Acosta-Pulido, J., et al. 2009, A\& A, 507, 881

Sipos, N., Kóspál, Á. in prep. 